SpringerBriefs in Electrical and Computer Engineering 
More information about this series at http://www.springer.com/series/10059 
Jiannong Cao $\cdot$ Xuefeng Liu

\section{Wireless Sensor Networks for Structural Health Monitoring}

望 Springer 
Jiannong Cao

Hong Kong Polytechnic University

Kowloon

Hong Kong
Xuefeng Liu

Hong Kong Polytechnic University

Kowloon

Hong Kong

ISSN 2191-8112

ISSN 2191-8120 (electronic)

SpringerBriefs in Electrical and Computer Engineering

ISBN 978-3-319-29032-4

ISBN 978-3-319-29034-8 (eBook)

DOI 10.1007/978-3-319-29034-8

\section{Library of Congress Control Number: 2016930052}

(C) The Author(s) 2016

This work is subject to copyright. All rights are reserved by the Publisher, whether the whole or part of the material is concerned, specifically the rights of translation, reprinting, reuse of illustrations, recitation, broadcasting, reproduction on microfilms or in any other physical way, and transmission or information storage and retrieval, electronic adaptation, computer software, or by similar or dissimilar methodology now known or hereafter developed.

The use of general descriptive names, registered names, trademarks, service marks, etc. in this publication does not imply, even in the absence of a specific statement, that such names are exempt from the relevant protective laws and regulations and therefore free for general use.

The publisher, the authors and the editors are safe to assume that the advice and information in this book are believed to be true and accurate at the date of publication. Neither the publisher nor the authors or the editors give a warranty, express or implied, with respect to the material contained herein or for any errors or omissions that may have been made.

Printed on acid-free paper

This Springer imprint is published by SpringerNature

The registered company is Springer International Publishing AG Switzerland 


\section{Preface}

\section{The Motivation for This Book}

The past decade has witnessed the emergence of applying wireless sensor networks (WSNs) to monitor the healthy condition of civil infrastructures. Compared to conventional wire-based structural healthy monitoring (SHM) systems, a WSN-based SHM system has the advantages of low cost, ease of deployment, and can obtain fine-grained information about structure's condition.

The main motivation for offering this book stems from the observation that, although many WSN-based SHM systems have been deployed, some real requirements in SHM have not been fully addressed. SHM applications have some distinct features from traditional WSN applications like environmental monitoring. Thus many widely adopted techniques in conventional applications of WSNs, like the event-triggered wake-up, in-network processing, fault-tolerance, cannot be directly applied for SHM applications. In this book, we first give a review of existing WSN-based SHM systems, and then introduce our WSN-based platform called SenetSHM. SenetSHM adopts many techniques that are specially designed to address the unique features of SHM applications. We share our experiences by stepping from the hardware and software design, to in-field experiments of the SenetSHM.

\section{What This Book Is About}

This book provides comprehensive coverage and detailed insights into the emerging area of using WSNs for SHM. It helps the readers to understand the specific requirements of SHM applications from other traditional WSN applications, and how these requirements are addressed using a series of systematic approaches. Therefore, it can be seen as a textbook as well as a practical guide for the reader: 
- To understand the state-of-the-art technologies in domain-specific applications of WSNs like SHM.

- To learn about the methodologies of how to address the specific requirements for a WSN application. In particular, we provide a guideline for problem formulation, problem solving, and share our experiences and lessons learned from our practices in implementing the SenetSHM.

\section{How This Book Is Organized}

This book is divided into seven chapters.

Chapter 1: Introduction. In this chapter, we introduce the background of SHM, some of the existing SHM systems, followed by the challenges and issues in developing the relevant technologies associated with WSN-based SHM systems.

Chapter 2: The Requirements and Design of Wireless Sensor Nodes for SHM. This chapter gives the requirements of SHM applications and an overview of our hardware and software design of wireless sensor nodes.

Chapter 3: Network-Wide and Reliable Event-Triggered Wakeup in WSNs. Wake-up scheduling is an important approach to save energy in WSNs. However, the requirements of wake-up in SHM are different from other applications of WSNs. This chapter provides the details of how to realize network-wide and reliable event-triggered wakeup in WSN-based SHM applications.

Chapter 4: Design of Distributed SHM Algorithms Within Wsns: A Cluster-Based Approach. Another important approach to address the resource-limited WSNs is to embed SHM algorithms within the network. However, typical SHM algorithms are centralized, computationally intensive, and are not easy to be distributed within a network. This chapter provides one typical approach, clustering, to design distributed versions of SHM algorithms.

Chapter 5: Design of Distributed SHM Algorithms Within WSNs: A Networked-Computing Approach. Although the cluster-based approach proposed in Chap. 4 is simple, the accuracy of damage detection obtained may not be guaranteed to be comparable with the centralized one. The goal of this chapter is to design a distributed SHM algorithm which is able to achieve the same accuracy as the centralized counterpart but uses much less wireless transmission cost. We believe the proposed schemes in these two chapters can serve as a guideline for designing distributed SHM algorithms in WSNs.

Chapter 6: Realizing Fault-Tolerant SHM in WSNs. It is well recognized that low cost wireless sensor nodes are likely to exhibit different types of faults, leading to downgrades of system performance. Existing fault-tolerance schemes usually cannot work well in SHM applications because SHM applications are generally data intensive. This chapter provides a series of systematic approaches to address the fault-tolerance issues in a WSN-based SHM system.

Chapter 7: Conclusion and Future Trends. This chapter provides the conclusions of this book. 


\section{Acknowledgments}

The authors are deeply grateful to the research staff and students in our research group for their hard work in carrying out the ITF project "A Versatile Wireless Sensor Network Platform for Structural Health Monitoring," and the Hong Kong PolyU Niche Area project "Structural Health Monitoring using Wireless Sensor Network." We thank our project group members, including Mr. Yang Liu, Dr. Md Zakirul Alam Bhuiyan, Dr. Chao Yang, and Mrs. Qionglei Hu. We also express our thanks to Prof. Wenzhan Song and Dr. Shaojie Tang for their invaluable advice throughout this research. The financial support from the Innovation and Technology Fund (ITF/39209) of the government of Hong Kong Special Administrative Region, and the NSF of China with Grant 61332004 and 61572218 is greatly appreciated. 


\section{Contents}

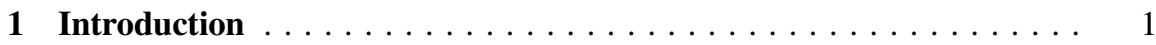

1.1 Structural Health Monitoring and Wire-Based Monitoring Systems . . . . . . . . . . . . . . . . . . 1

1.2 Structural Health Monitoring Using Wireless Sensor Networks ... 3

References ......................... 6

2 Requirements, Challenges, and Summary of Hardware

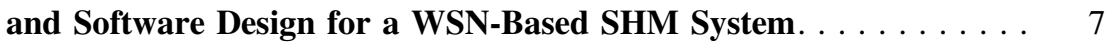

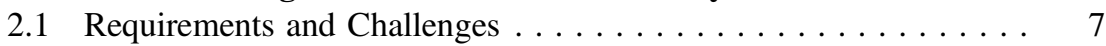

2.2 Hardware Design. . . . . . . . . . . . . . . . . . . 8

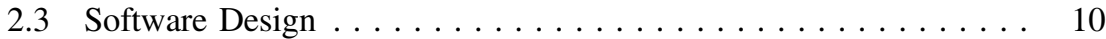

References ......................... 11

3 Enabling Network-Wide and Event-Triggered Wakeup . . . . . . . 13

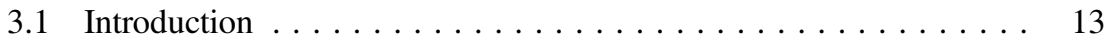

3.2 Related Works. . . . . . . . . . . . . . . . . . . 14

3.3 Preliminaries....................... 16

3.3.1 Two Wakeup Units and the Chain-Reaction Wakeup . . . . 16

3.3.2 Event, False Positive, and False Negative. . . . . . . . . . 17

3.3.3 Wakeup Graph and Wakeup Delay . . . . . . . . . . . 19

3.4 Sentry Node Placement Problem (SNPP) $\ldots \ldots \ldots \ldots \ldots \ldots \ldots .22$

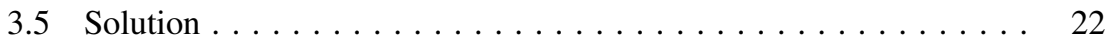

3.6 Simulation ........................ 24

3.6.1 Spanning Tree-Based Simulation . . . . . . . . . . . . 25

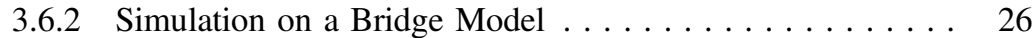

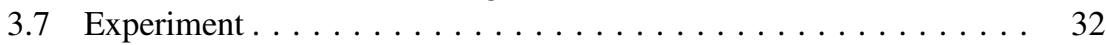

References ......................... 34

4 Design of Distributed SHM Algorithms Within WSNs-A

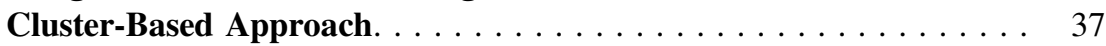

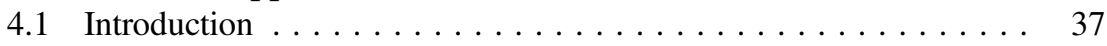

4.2 Preliminary: Modal Parameters and the ERA. . . . . . . . . 39 
4.3 Cluster-Based Modal Analysis and Its Energy Consumption . . . . . 42

4.4 Determination of Global Mode Shapes Through Mode Shape Assembling . . . . . . . . . . . . . . . . . . 45

4.5 Optimal Clustering. . . . . . . . . . . . . . . . 47

4.5.1 Proposed Methods for Energy Efficient Clustering . . . . . . . 48

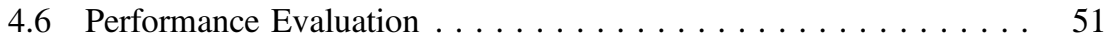

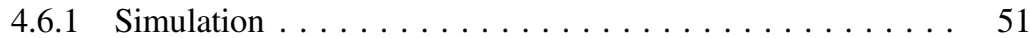

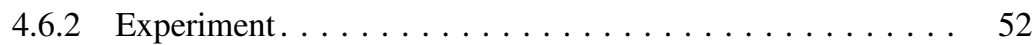

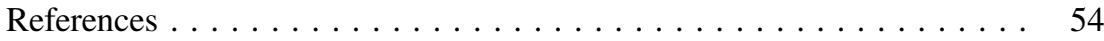

5 Design of Distributed SHM Algorithms Within WSNs-A

Networked-Computing Approach . . . . . . . . . . . . . . 57

5.1 Design Objectives and the Organization of This Chapter. . . . . . 57

5.2 Modification 1: Calculating the Markov Parameters in a Distributed Manner . . . . . . . . . . . . . . . . 58

5.3 Modification 2: Incremental SVD of the Hankel Matrix . . . . . . . . . 59

5.4 Modification 3: Incremental SVD Updating in a WSN . . . . . . . . 62

5.4.1 Updating the SVD Along a Hamiltonian Path in the Network . . . . . . . . . . . . . . . . . 63

5.4.2 Updating the SVD Along a Route in the Minimum-Connected Dominating Set (MCDS) . . . 64

5.4.3 Modification 5: Updating the SVD Along the Shortest Path Tree ......................... 67

5.5 Summary of Communication and Computation Cost of the Proposed Methods . . . . . . . . . . . . . . 71

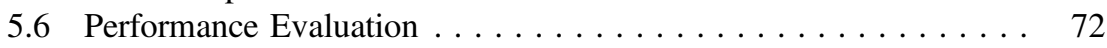

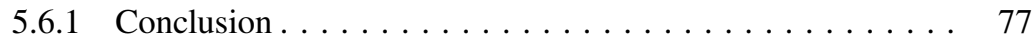

References . . . . . . . . . . . . . . . . . . . . . . . . . 77

6 Realizing Fault-Tolerant SHM in WSNs . . . . . . . . . . . . . . 79

6.1 Introduction . . . . . . . . . . . . . . . . . . . . . . . . . . . 79

6.2 Related Works. . . . . . . . . . . . . . . . . . . 81

6.3 FTED: The Fault-Tolerant Event Detection Scheme

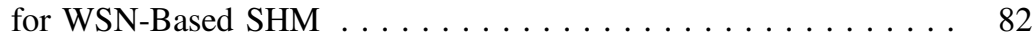

6.3.1 Natural Frequency and Mode Shape . . . . . . . . . . . . 83

6.3.2 Cluster-Based Natural Frequency Identification . . . . . . . . 84

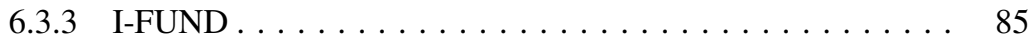

6.3 .4 Identification of Mode Shape . . . . . . . . . . . . . . . 91

6.4 Simulation . . . . . . . . . . . . . . . . . . . . 92

6.4.1 The Performance of I-FUND with ROD Through

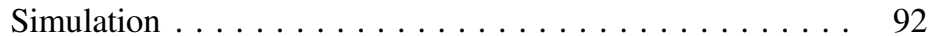

6.5 Experiment on a Lab Structure . . . . . . . . . . . . . . . . 95

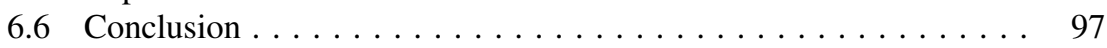

References ............................. 97

7 Conclusions . . . . . . . . . . . . . . . . . . . . . . . . . . 99 